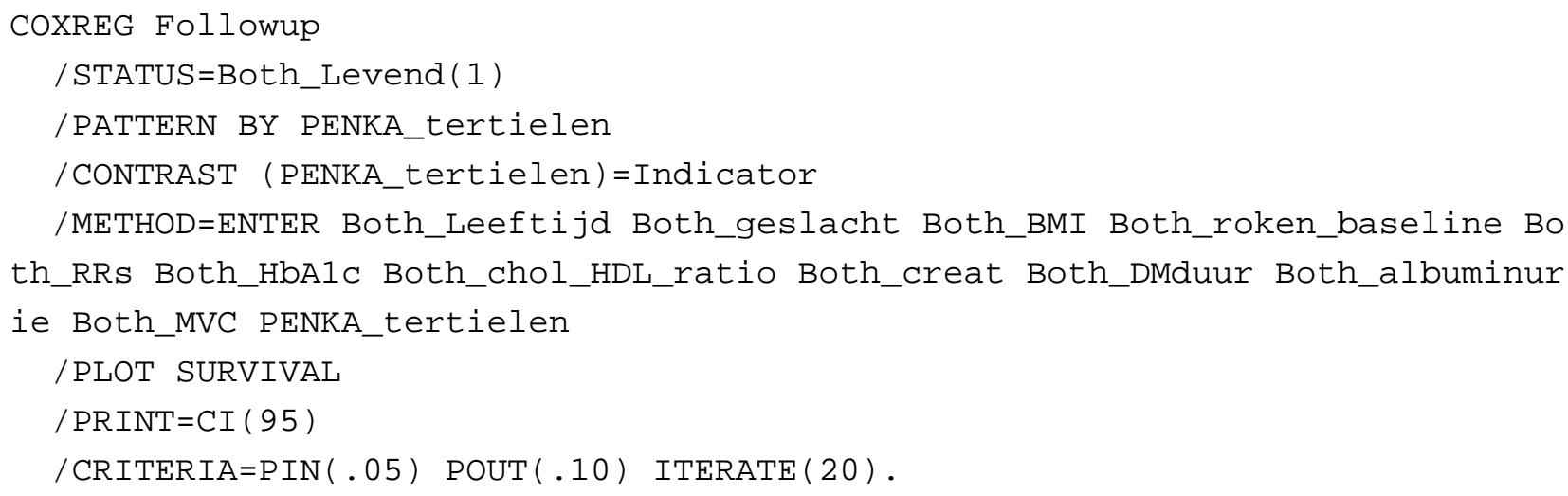

\title{
Cox Regression
}

\section{Notes}

\begin{tabular}{|c|c|c|}
\hline \multicolumn{2}{|l|}{ Output Created } & 17-sep-2014 12:24:29 \\
\hline \multicolumn{3}{|l|}{ Comments } \\
\hline \multirow[t]{6}{*}{ Input } & Data & 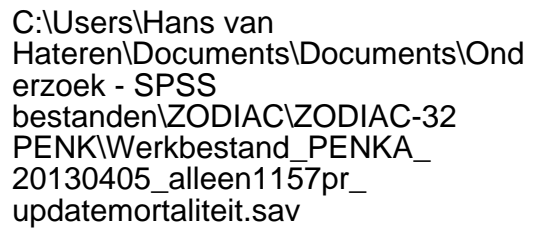 \\
\hline & Active Dataset & DataSet1 \\
\hline & Filter & $<$ none $>$ \\
\hline & Weight & $<$ none $>$ \\
\hline & Split File & $<$ none $>$ \\
\hline & $\begin{array}{l}N \text { of Rows in Working } \\
\text { Data File }\end{array}$ & 1157 \\
\hline Missing Value Handling & Definition of Missing & $\begin{array}{l}\text { User-defined missing values are } \\
\text { treated as missing. }\end{array}$ \\
\hline Syntax & & $\begin{array}{l}\text { COXREG Followup } \\
\text { /STATUS=Both_Levend(1) } \\
\text { /PATTERN BY PENKA_tertielen } \\
\text { /CONTRAST (PENKA_tertielen) } \\
\text { =Indicator } \\
\text { /METHOD=ENTER Both_Leeftijd } \\
\text { Both_geslacht Both_BMI } \\
\text { Both_roken_baseline Both_RRs } \\
\text { Both_HbA1c Both_chol_HDL_ratio } \\
\text { Both_creat Both_DMduur } \\
\text { Both_albuminurie Both_MVC } \\
\text { PENKA_tertielen } \\
\text { /PLOTSURVIVAL } \\
\text { /PRINT=CI(95) } \\
\text { /CRITERIA=PIN(.05) POUT(.10) } \\
\text { ITERATE(20). }\end{array}$ \\
\hline \multirow[t]{2}{*}{ Resources } & Processor Time & $00: 00: 00,406$ \\
\hline & Elapsed Time & $00: 00: 00,416$ \\
\hline
\end{tabular}

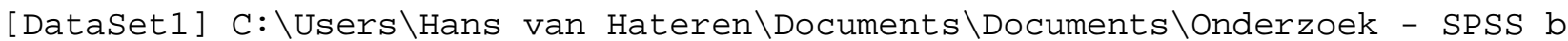
estanden \ZODIAC \ZODIAC-32 PENK\Werkbestand_PENKA_20130405_alleen1157pr_upda temortaliteit.sav 


\section{Case Processing Summary}

\begin{tabular}{|c|c|c|c|}
\hline & & $\mathrm{N}$ & Percent \\
\hline \multirow{3}{*}{$\begin{array}{l}\text { Cases available in } \\
\text { analysis }\end{array}$} & Event $^{\mathrm{a}}$ & 525 & $45,4 \%$ \\
\hline & Censored & 632 & $54,6 \%$ \\
\hline & Total & 1157 & $100,0 \%$ \\
\hline \multirow[t]{4}{*}{ Cases dropped } & Cases with missing values & 0 &, $0 \%$ \\
\hline & Cases with negative time & 0 &, $0 \%$ \\
\hline & $\begin{array}{l}\text { Censored cases before } \\
\text { the earliest event in a } \\
\text { stratum }\end{array}$ & 0 &, $0 \%$ \\
\hline & Total & 0 &, $0 \%$ \\
\hline Total & & 1157 & $100,0 \%$ \\
\hline
\end{tabular}

a. Dependent Variable: Followup

Categorical Variable Codings ${ }^{b}$

\begin{tabular}{|ll|r|r|r|}
\hline & & Frequency & (1) & (2) \\
\hline PENKA_tertielen $^{\mathrm{a}}$ & 1 & 385 & 1 & 0 \\
& 2 & 389 & 0 & 1 \\
& 3 & 383 & 0 & 0 \\
\hline
\end{tabular}

a. Indicator Parameter Coding

b. Category variable: PENKA_tertielen (penka log verdeeld in tertielen)

\section{Block 0: Beginning Block}

\section{Omnibus Tests of}

Model Coefficients

-2 Log Likelihood

7004,412

\section{Block 1: Method = Enter}

Omnibus Tests of Model Coefficients ${ }^{a}$

\begin{tabular}{|c|r|r|r|r|r|r|}
\hline \multirow{2}{*}{$\begin{array}{c}-2 \text { Log } \\
\text { Likelihood }\end{array}$} & \multicolumn{3}{|c|}{ Overall (score) } & \multicolumn{3}{c|}{ Change From Previous Step } \\
\cline { 2 - 7 } & Chi-square & $\mathrm{df}$ & Sig. & Chi-square & \multicolumn{1}{c|}{$\mathrm{df}$} & Sig. \\
\hline 6367,634 & 611,068 & 13 &, 000 & 636,778 & 13 &, 000 \\
\hline
\end{tabular}

a. Beginning Block Number 1. Method = Enter

\section{Omnibus Tests of Model Coefficients ${ }^{a}$}

\begin{tabular}{|r|r|r|}
\hline \multicolumn{3}{|c|}{ Change From Previous Block } \\
\hline Chi-square & df & Sig. \\
\hline 636,778 & 13 &, 000 \\
\hline
\end{tabular}

a. Beginning Block Number 1. Method = Enter 
Variables in the Equation

\begin{tabular}{|l|r|r|r|r|r|r|}
\hline & \multicolumn{1}{|c|}{ B } & \multicolumn{1}{c|}{ SE } & \multicolumn{1}{c|}{ Wald } & df & \multicolumn{1}{c|}{ Sig. } & \multicolumn{1}{c|}{ Exp(B) } \\
\hline Both_Leeftijd &, 103 &, 006 & 313,715 & 1 &, 000 & 1,108 \\
Both_geslacht &,- 103 &, 102 & 1,017 & 1 &, 313 &, 902 \\
Both_BMI &, 002 &, 011 &, 032 & 1 &, 858 & 1,002 \\
Both_roken_baseline &, 682 &, 119 & 33,131 & 1 &, 000 & 1,979 \\
Both_RRs &,- 004 &, 002 & 4,381 & 1 &, 036 &, 996 \\
Both_HbA1c &, 094 &, 037 & 6,504 & 1 &, 011 & 1,099 \\
Both_chol_HDL_ratio &,- 008 &, 032 &, 058 & 1 &, 810 &, 992 \\
Both_creat &, 009 &, 002 & 17,606 & 1 &, 000 & 1,009 \\
Both_DMduur &, 016 &, 006 & 6,890 & 1 &, 009 & 1,016 \\
Both_albuminurie &, 550 &, 094 & 34,083 & 1 &, 000 & 1,734 \\
Both_MVC &, 408 &, 092 & 19,846 & 1 &, 000 & 1,504 \\
PENKA_tertielen & & &, 246 & 2 &, 884 &, 641 \\
PENKA_tertielen(1) &,- 058 &, 124 &, 218 & 1 &, 944 \\
PENKA_tertielen(2) &,- 010 &, 112 &, 007 & 1 &, 932 &, 990 \\
\hline
\end{tabular}

Variables in the Equation

\begin{tabular}{|l|r|r|}
\hline \multirow{2}{*}{} & \multicolumn{2}{|c|}{$95,0 \%$ Cl for Exp(B) } \\
\cline { 2 - 3 } & \multicolumn{1}{|c|}{ Lower } & \multicolumn{1}{c|}{ Upper } \\
\hline Both_Leeftijd & 1,096 & 1,121 \\
Both_geslacht &, 738 & 1,102 \\
Both_BMI &, 981 & 1,023 \\
Both_roken_baseline & 1,568 & 2,496 \\
Both_RRs &, 991 & 1,000 \\
Both_HbA1c & 1,022 & 1,182 \\
Both_chol_HDL_ratio &, 932 & 1,056 \\
Both_creat & 1,005 & 1,014 \\
Both_DMduur & 1,004 & 1,027 \\
Both_albuminurie & 1,441 & 2,086 \\
Both_MVC & 1,257 & 1,800 \\
PENKA_tertielen & & \\
PENKA_tertielen(1) &, 740 & 1,204 \\
PENKA_tertielen(2) &, 795 & 1,234 \\
\hline
\end{tabular}


Covariate Means and Pattern Values

\begin{tabular}{|l|r|r|r|r|}
\hline & \multirow{2}{*}{ Mean } & \multicolumn{3}{|c|}{ Pattern } \\
\cline { 3 - 5 } & 66,602 & 66,602 & \multicolumn{1}{|c|}{2} & \multicolumn{1}{c|}{3} \\
\hline Both_Leeftijd & 1,551 & 1,551 & 1,551 & 1,551 \\
Both_geslacht & 29,205 & 29,205 & 29,205 & 29,205 \\
Both_BMI & 1,188 & 1,188 & 1,188 & 1,188 \\
Both_roken_baseline & 152,038 & 152,038 & 152,038 & 152,038 \\
Both_RRs & 7,245 & 7,245 & 7,245 & 7,245 \\
Both_HbA1c & 4,898 & 4,898 & 4,898 & 4,898 \\
Both_chol_HDL_ratio & 94,838 & 94,838 & 94,838 & 94,838 \\
Both_creat & 6,582 & 6,582 & 6,582 & 6,582 \\
Both_DMduur & 1,392 & 1,392 & 1,392 & 1,392 \\
Both_albuminurie &, 356 &, 356 &, 356 &, 356 \\
Both_MVC &, 333 & 1,000 &, 000 &, 000 \\
PENKA_tertielen(1) &, 336 &, 000 & 1,000 &, 000 \\
PENKA_tertielen(2) & &
\end{tabular}

\section{Survival Function at mean of covariates}

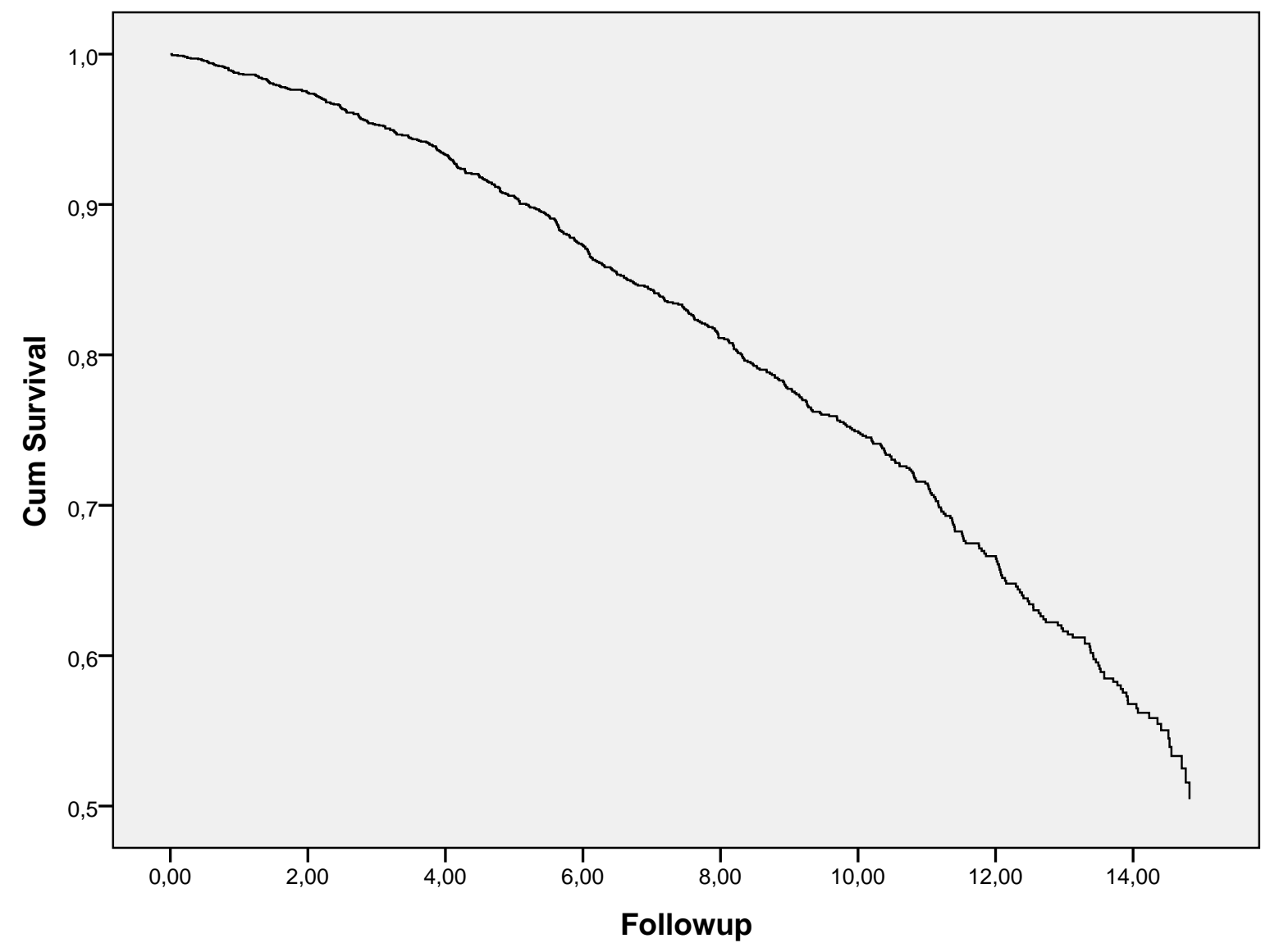



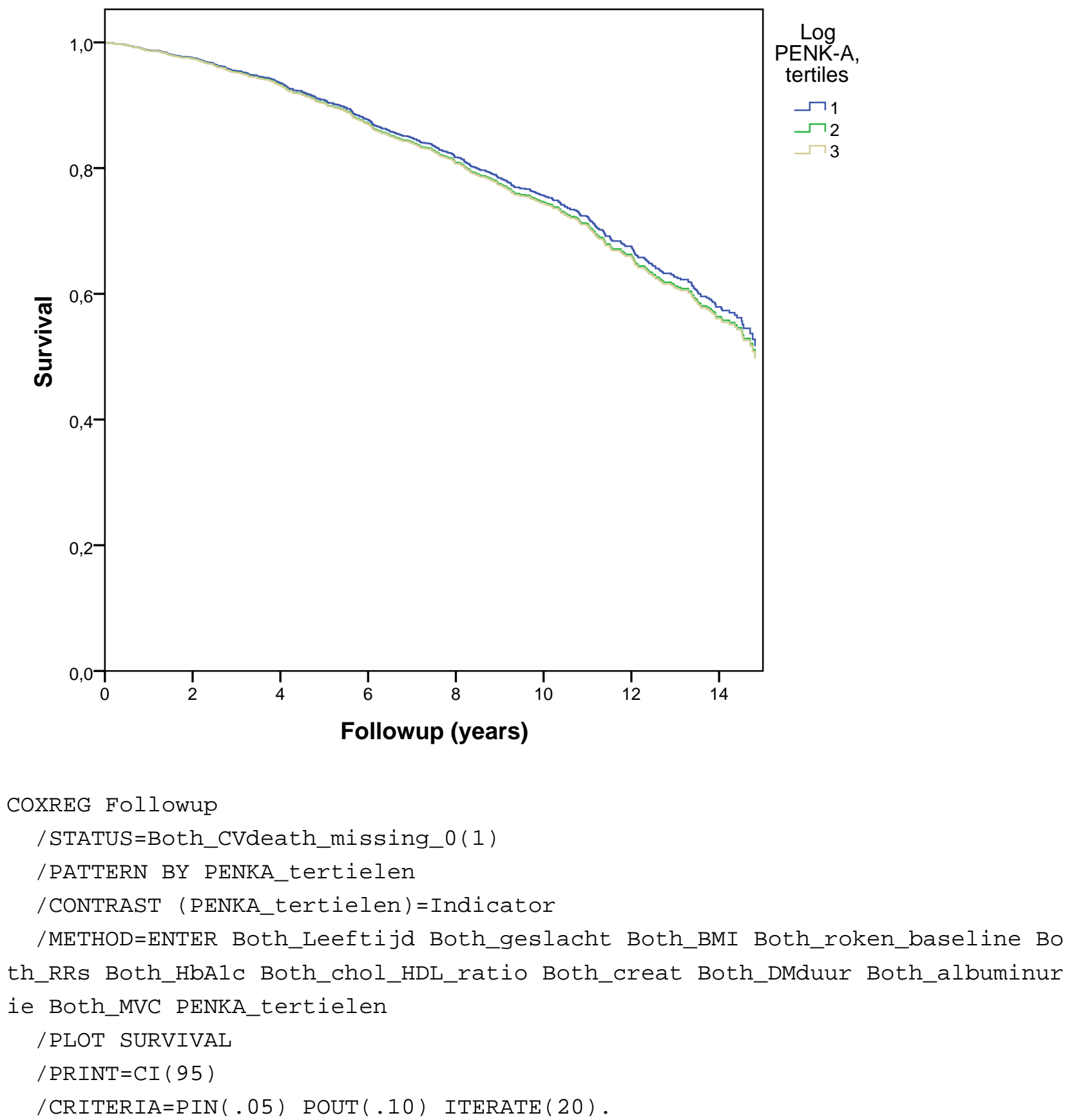

\section{Cox Regression}


Notes

\begin{tabular}{|c|c|c|}
\hline Output Created & & \multirow[t]{2}{*}{ 17-sep-2014 12:24:41 } \\
\hline Comments & & \\
\hline \multirow[t]{6}{*}{ Input } & Data & 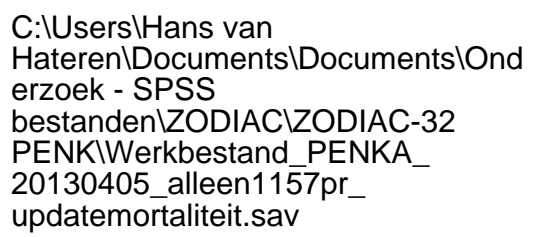 \\
\hline & Active Dataset & DataSet1 \\
\hline & Filter & $<$ none $>$ \\
\hline & Weight & $<$ none $>$ \\
\hline & Split File & $<$ none $>$ \\
\hline & $\begin{array}{l}\mathrm{N} \text { of Rows in Working } \\
\text { Data File }\end{array}$ & 1157 \\
\hline Missing Value Handling & Definition of Missing & $\begin{array}{l}\text { User-defined missing values are } \\
\text { treated as missing. }\end{array}$ \\
\hline Syntax & & $\begin{array}{l}\text { COXREG Followup } \\
\text { /STATUS=Both_CVdeath_missing_0 } \\
\text { (1) } \\
\text { /PATTERN BY PENKA_tertielen } \\
\text { /CONTRAST (PENKA_tertielen) } \\
\text { =Indicator } \\
\text { /METHOD=ENTER Both_Leeftijd } \\
\text { Both_geslacht Both_BMI } \\
\text { Both_roken_baseline Both_RRs } \\
\text { Both_HbA1c Both_chol_HDL_ratio } \\
\text { Both_creat Both_DMduur } \\
\text { Both_albuminurie Both_MVC } \\
\text { PENKA_tertielen } \\
\text { /PLOTSURVIVAL } \\
\text { /PRINT=CI(95) } \\
\text { /CRITERIA=PIN(.05) POUT(.10) } \\
\text { ITERATE(20). }\end{array}$ \\
\hline \multirow[t]{2}{*}{ Resources } & Processor Time & $00: 00: 00,343$ \\
\hline & Elapsed Time & 00:00:00,337 \\
\hline
\end{tabular}

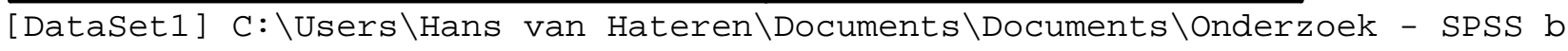
estanden \ZODIAC $\backslash$ ZODIAC-32 PENK \Werkbestand_PENKA_20130405_alleen1157pr_upda temortaliteit.sav 


\section{Case Processing Summary}

\begin{tabular}{|c|c|c|c|}
\hline & & $\mathrm{N}$ & Percent \\
\hline \multirow{3}{*}{$\begin{array}{l}\text { Cases available in } \\
\text { analysis }\end{array}$} & Event $^{\mathrm{a}}$ & 224 & $19,4 \%$ \\
\hline & Censored & 933 & $80,6 \%$ \\
\hline & Total & 1157 & $100,0 \%$ \\
\hline \multirow[t]{4}{*}{ Cases dropped } & Cases with missing values & 0 &, $0 \%$ \\
\hline & Cases with negative time & 0 &, $0 \%$ \\
\hline & $\begin{array}{l}\text { Censored cases before } \\
\text { the earliest event in a } \\
\text { stratum }\end{array}$ & 0 &, $0 \%$ \\
\hline & Total & 0 &, $0 \%$ \\
\hline Total & & 1157 & $100,0 \%$ \\
\hline
\end{tabular}

a. Dependent Variable: Followup

Categorical Variable Codings ${ }^{b}$

\begin{tabular}{|ll|r|r|r|}
\hline & & Frequency & (1) & (2) \\
\hline PENKA_tertielen $^{\mathrm{a}}$ & 1 & 385 & 1 & 0 \\
& 2 & 389 & 0 & 1 \\
& 3 & 383 & 0 & 0 \\
\hline
\end{tabular}

a. Indicator Parameter Coding

b. Category variable: PENKA_tertielen (penka log verdeeld in tertielen)

\section{Block 0: Beginning Block}

\section{Omnibus Tests of}

Model Coefficients

-2 Log Likelihood

3004,647

\section{Block 1: Method = Enter}

Omnibus Tests of Model Coefficients ${ }^{a}$

\begin{tabular}{|c|r|r|r|r|r|r|}
\hline \multirow{2}{*}{$\begin{array}{c}-2 \text { Log } \\
\text { Likelihood }\end{array}$} & \multicolumn{3}{|c|}{ Overall (score) } & \multicolumn{3}{c|}{ Change From Previous Step } \\
\cline { 2 - 7 } & Chi-square & df & Sig. & Chi-square & \multicolumn{1}{c|}{ df } & Sig. \\
\hline 2663,253 & 365,681 & 13 &, 000 & 341,394 & 13 &, 000 \\
\hline
\end{tabular}

a. Beginning Block Number 1. Method = Enter

\section{Omnibus Tests of Model Coefficients ${ }^{a}$}

\begin{tabular}{|r|r|r|}
\hline \multicolumn{3}{|c|}{ Change From Previous Block } \\
\hline Chi-square & df & Sig. \\
\hline 341,394 & 13 &, 000 \\
\hline
\end{tabular}

a. Beginning Block Number 1. Method = Enter 
Variables in the Equation

\begin{tabular}{|l|r|r|r|r|r|r|}
\hline & \multicolumn{1}{|c|}{ B } & \multicolumn{1}{|c|}{ SE } & \multicolumn{1}{c|}{ Wald } & df & \multicolumn{1}{c|}{ Sig. } & Exp(B) \\
\hline Both_Leeftijd &, 089 &, 009 & 100,835 & 1 &, 000 & 1,094 \\
Both_geslacht &, 004 &, 154 &, 001 & 1 &, 978 & 1,004 \\
Both_BMI &,- 001 &, 016 &, 006 & 1 &, 938 &, 999 \\
Both_roken_baseline &, 907 &, 176 & 26,568 & 1 &, 000 & 2,477 \\
Both_RRs &,- 002 &, 003 &, 311 & 1 &, 577 &, 998 \\
Both_HbA1c &, 154 &, 055 & 7,735 & 1 &, 005 & 1,166 \\
Both_chol_HDL_ratio &,- 019 &, 048 &, 156 & 1 &, 693 &, 981 \\
Both_creat &, 015 &, 003 & 27,357 & 1 &, 000 & 1,015 \\
Both_DMduur &, 020 &, 008 & 5,665 & 1 &, 017 & 1,020 \\
Both_albuminurie &, 693 &, 148 & 22,017 & 1 &, 000 & 2,000 \\
Both_MVC &, 667 &, 142 & 22,219 & 1 &, 000 & 1,949 \\
PENKA_tertielen & & & 4,119 & 2 &, 128 &, 044 \\
PENKA_tertielen(1) &,- 401 &, 199 & 4,064 & 1 &, 670 \\
PENKA_tertielen(2) &,- 190 &, 173 & 1,211 & 1 &, 271 &, 827 \\
\hline
\end{tabular}

Variables in the Equation

\begin{tabular}{|l|r|r|}
\hline \multirow{2}{*}{} & \multicolumn{2}{|c|}{$95,0 \% \mathrm{Cl}$ for Exp(B) } \\
\cline { 2 - 3 } & \multicolumn{1}{|c|}{ Lower } & \multicolumn{1}{c|}{ Upper } \\
\hline Both_Leeftijd & 1,075 & 1,113 \\
Both_geslacht &, 743 & 1,358 \\
Both_BMI &, 967 & 1,031 \\
Both_roken_baseline & 1,754 & 3,497 \\
Both_RRs &, 992 & 1,005 \\
Both_HbA1c & 1,047 & 1,300 \\
Both_chol_HDL_ratio &, 893 & 1,078 \\
Both_creat & 1,009 & 1,020 \\
Both_DMduur & 1,004 & 1,037 \\
Both_albuminurie & 1,497 & 2,671 \\
Both_MVC & 1,477 & 2,572 \\
PENKA_tertielen & & \\
PENKA_tertielen(1) &, 454 &, 989 \\
PENKA_tertielen(2) &, 590 & 1,160 \\
\hline
\end{tabular}


Covariate Means and Pattern Values

\begin{tabular}{|l|r|r|r|r|}
\hline & \multirow{2}{*}{ Mean } & \multicolumn{3}{|c|}{ Pattern } \\
\cline { 3 - 5 } & 66,602 & 66,602 & \multicolumn{1}{|c|}{2} & \multicolumn{1}{c|}{3} \\
\hline Both_Leeftijd & 1,551 & 1,551 & 1,551 & 1,551 \\
Both_geslacht & 29,205 & 29,205 & 29,205 & 29,205 \\
Both_BMI & 1,188 & 1,188 & 1,188 & 1,188 \\
Both_roken_baseline & 152,038 & 152,038 & 152,038 & 152,038 \\
Both_RRs & 7,245 & 7,245 & 7,245 & 7,245 \\
Both_HbA1c & 4,898 & 4,898 & 4,898 & 4,898 \\
Both_chol_HDL_ratio & 94,838 & 94,838 & 94,838 & 94,838 \\
Both_creat & 6,582 & 6,582 & 6,582 & 6,582 \\
Both_DMduur & 1,392 & 1,392 & 1,392 & 1,392 \\
Both_albuminurie &, 356 &, 356 &, 356 &, 356 \\
Both_MVC &, 333 & 1,000 &, 000 &, 000 \\
PENKA_tertielen(1) &, 336 &, 000 & 1,000 &, 000 \\
PENKA_tertielen(2) & &
\end{tabular}

\section{Survival Function at mean of covariates}

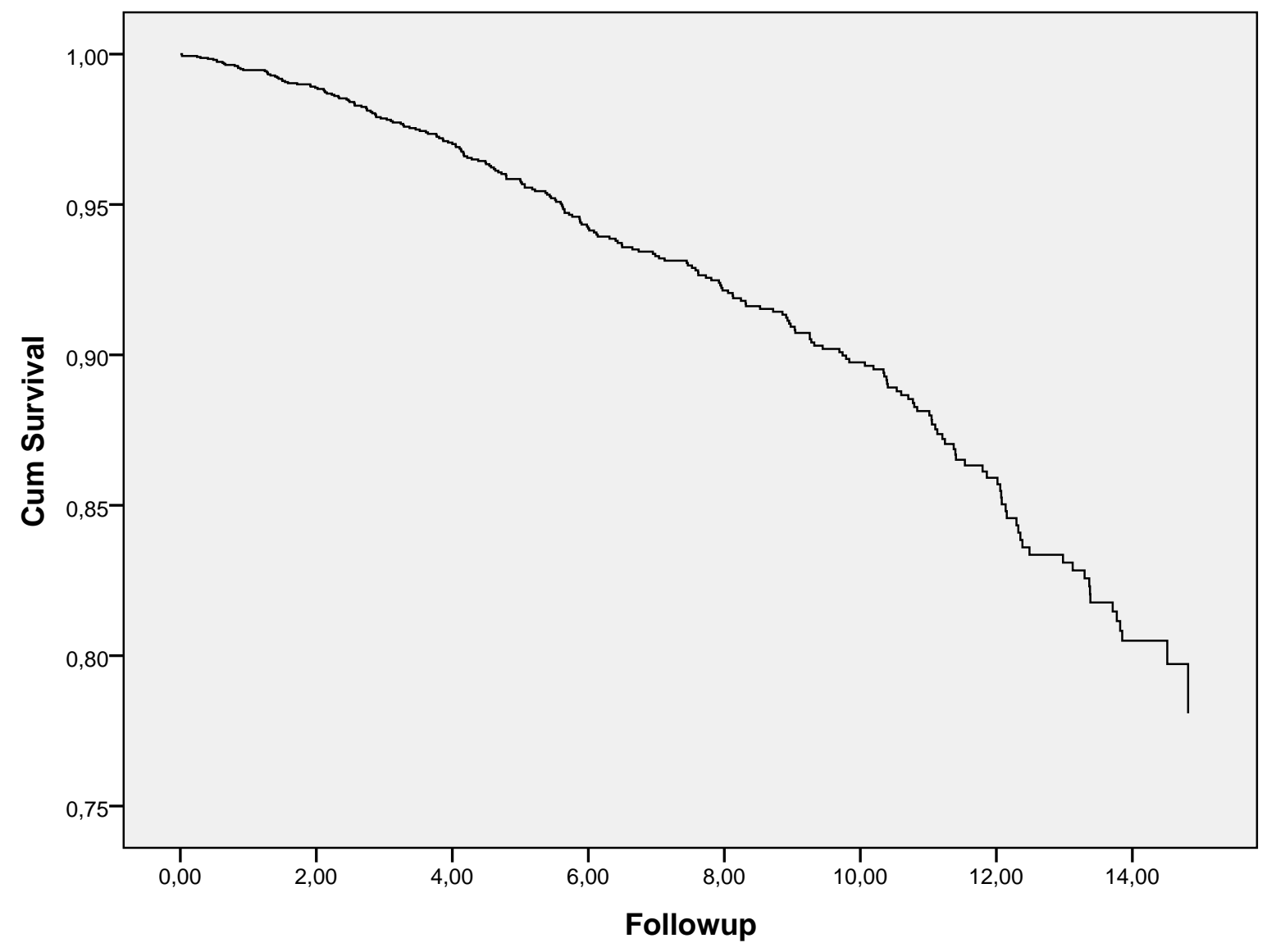




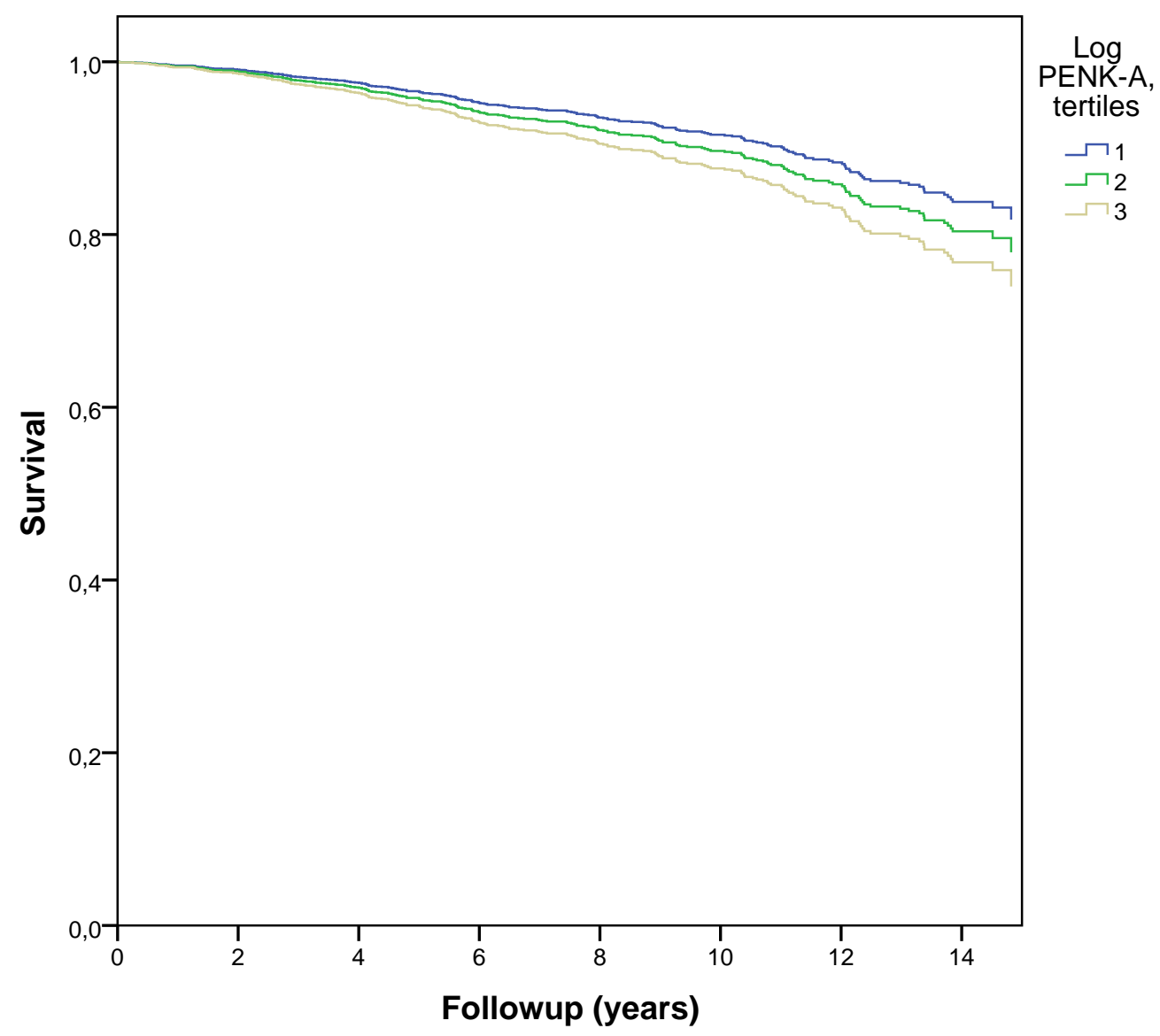

Page 10 